\title{
Cardiac biomarkers and bone fractures
}

Fragility-related fractures and cardiovascular disease are common conditions in old age, both contributing to impaired quality of life and higher risk of death in the elderly. In this issue of Heart, Johansson $e$ t $a l^{1}$ present results suggesting that these conditions may be more closely related than previously considered. The authors hypothesised that cardiovascular autonomic disorder and endothelial dysfunction, as reflected in serum biomarker levels, would be associated with fracture risk. Studying a subset of the Malmö Preventive Project, a population-based prospective cohort, they examined the association of four cardiovascular biomarkers with the risk of incident vertebral, pelvic and extremity fractures in 5415 community-dwelling subjects (mean age, $68.9 \pm 6.2$ years), followed during $8.1 \pm 2.9$ years. C-terminal pro-arginine vasopressin (CT-proAVP), C-terminal endothelin-1 precursor fragment (CT-proET-1), the mid-regional fragments of pro-adrenomedullin (MR-proADM) and pro-atrial natriuretic peptide (MR-proANP) were measured in fasting blood samples during re-screening examination in years 2002-2006. Information about fracture diagnoses and the date of death were retrieved from the Swedish National Hospital Discharge Register and the Swedish National Cause of Death Register. Elevated levels of MR-proADM and MR-proANP independently predicted fragility fractures in older adults, with a stronger association in men compared with women. In subjects with levels of all four biomarker in the top quartile, there was a 2- to 3-fold increase in risk of vertebral and femoral fractures. (figure 1)

In the companion editorial, Lewis and Szulc ${ }^{2}$ point out the strengths of the study including investigation of vascular biomarkers and clinically important fractures in a large well-powered cohort of both older men and women. However, they point out potential limitations; this is an observational study so causality cannot be established, several other factors related

${ }^{1}$ Internal Medicine, Universidade Federal de Minas Gerais, Belo Horizonte, Brazil

${ }^{2}$ Division of Cardiology, University of Washington, Seattle, Washington, USA

Correspondence to Professor Catherine M Otto, Division of Cardiology, University of Washington, SeattleWA 98195, USA ; cmotto@uw.edu

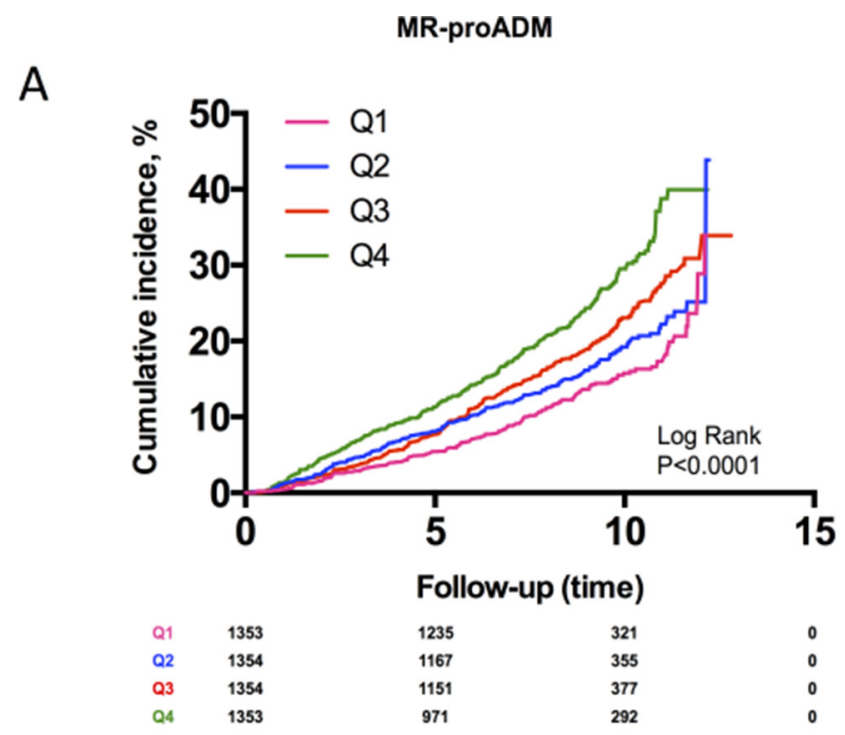

Figure 1 Kaplan-Meier curves for cumulative incidence of fragility fractures by quartiles of MRproADMCT-proAVP. MR-proADM, mid-regional pro-adrenomedullin.

to fall risk were not assessed, and only a few cardiac biomarkers were measured. Thus, this data should not yet change clinical practice. Even so, the association between cardiovascular disease (CVD) and fracture risk deserves closer study. "In particular, further research in this area may identify novel non-bone-related treatments for reducing the burden of minimal trauma fractures in older men, an often overlooked and undertreated group.'

Occupational exposure to chemicals has rarely been studied despite the known cardiotoxic properties of many of these agents. In order to fill this knowledge gap, Bulka and colleagues ${ }^{3}$ evaluated the association of self-reported occupational exposure and the prevalence of CVD in a cross-sectional study of over 7000 employed Hispanic/Latino adults in the USA. The reported prevalence of current occupational exposure was $6.5 \%$ for organic solvents, $8.5 \%$ for metals and $4.7 \%$ for pesticides. Overall, 6.1\% had CVD including coronary heart disease $(4.3 \%)$ cerebrovascular disease $(1.0 \%)$, heart failure $(0.8 \%)$ and atrial fibrillation $(0.7 \%)$. The prevalence ratio for any CVD was higher by 2 -fold $(2.18,95 \% \mathrm{CI}$ 1.34 to 3.55 ) for pesticide exposure even after adjustment for sociodemographic, acculturation, lifestyle and occupational characteristics. (figure 2) There was an almost fourfold $(3.78,95 \% \mathrm{CI} 1.24$ to 11.46) greater prevalence of atrial fibrillation in subjects reporting metal exposures. No CVD associations were observed for organic solvent exposures.

In an editorial, Broberg ${ }^{4}$ discuss the possible mechanisms of occupational exposure to chemicals and CVD risk. "Many metals are strong inducers of oxidative stress which may result in CVD either directly or via increased inflammation. Association between pesticides and CVD are less well studied, and although some pesticides cause oxidative stress, other mechanisms are likely to be important.' She concludes: "Exposure to metals and pesticides is common worldwide, and this study highlights the need to better understand the risks that these exposures cause, and to limit exposure in the workplace, thus promoting cardiac health.'

Quality improvement (QI) interventions are often challenging, and the effect of these efforts can be difficult to measure. In a structured systematic review of studies evaluating the effect of in-hospital QI for patients with heart failure, Agarwal et $a l^{5}$ identified 14 relevant randomised trials using a variety of QI interventions. Although QI intervention was associated with favourable trends for medical therapy and reduced readmission rates in few trials, overall, there was no consistent 


\section{Pesticides}

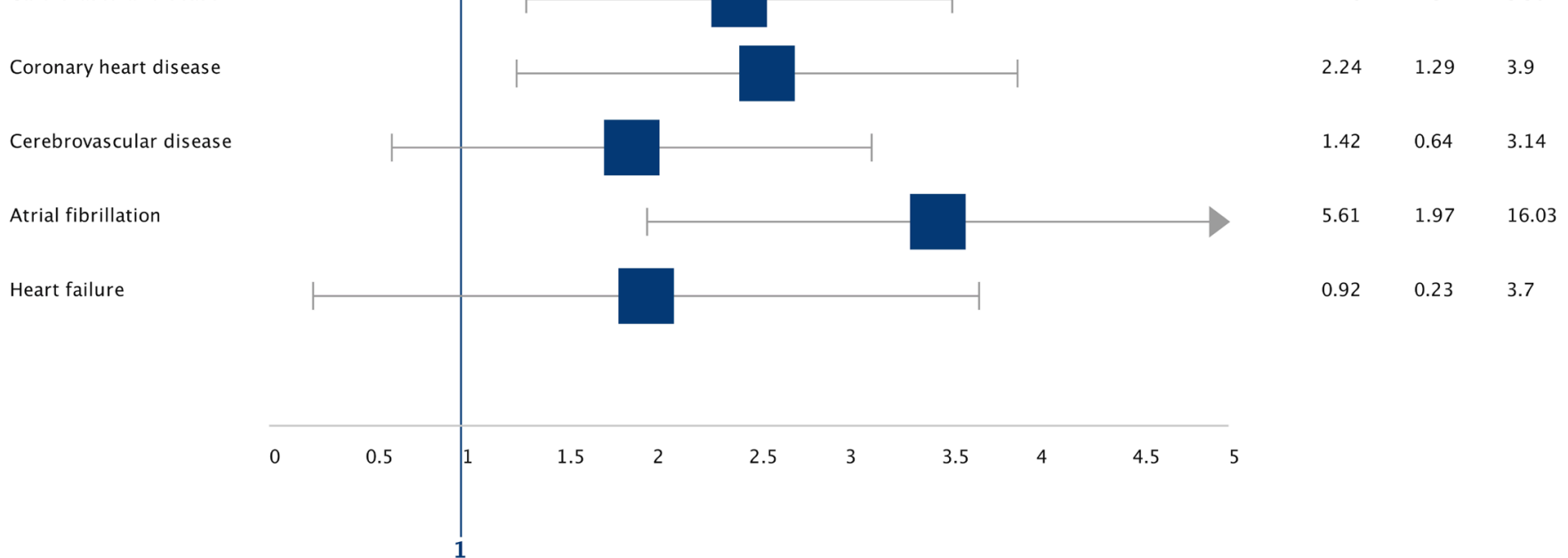

Figure 2 Prevalence ratios with $95 \% \mathrm{Cl}$ for current occupational exposure to pesticides and cardiovascular disease after adjustment for age, gender, field centre, Hispanic/Latino background, current health insurance status, cigarette use, alcohol use level, years of residential duration in the USA, employment status, physical activity level, alternative healthy eating index, Short Acculturation Scale for Hispanics - language subscale, hypertension, hypercholesterolaemia, body mass index and diabetes mellitus. LCL, lower confidence limit; UCL, upper confidence limit.

effect of QI on 30 day mortality, hospital length of stay or health-related quality of life. These disappointing results might be related to the poor quality of evidence for the primary and secondary outcomes, suggesting that future research would provide more reliable estimates for any benefit of QI for patients admitted with heart failure.
Ezekowitz ${ }^{6}$ puts this study in context and points out that "The connectivity of quality improvement of inpatient to the outpatient environment remains a key gap (and often chasm) that needs a deep local understanding to best improve the transmission of quality.' (figure 3)

Management of heart failure in the outpatient setting is even more difficult than in the hospital due to the multiple clinics and clinicians involved, lack of urgency, and absence of systems to coordinate patient care. He suggest keys to designing effective QI: "Establish a culture of innovation, quality and teamwork; prioritise the QI based on patient needs and outcomes; collect data and focus measurement activity; be transparent and

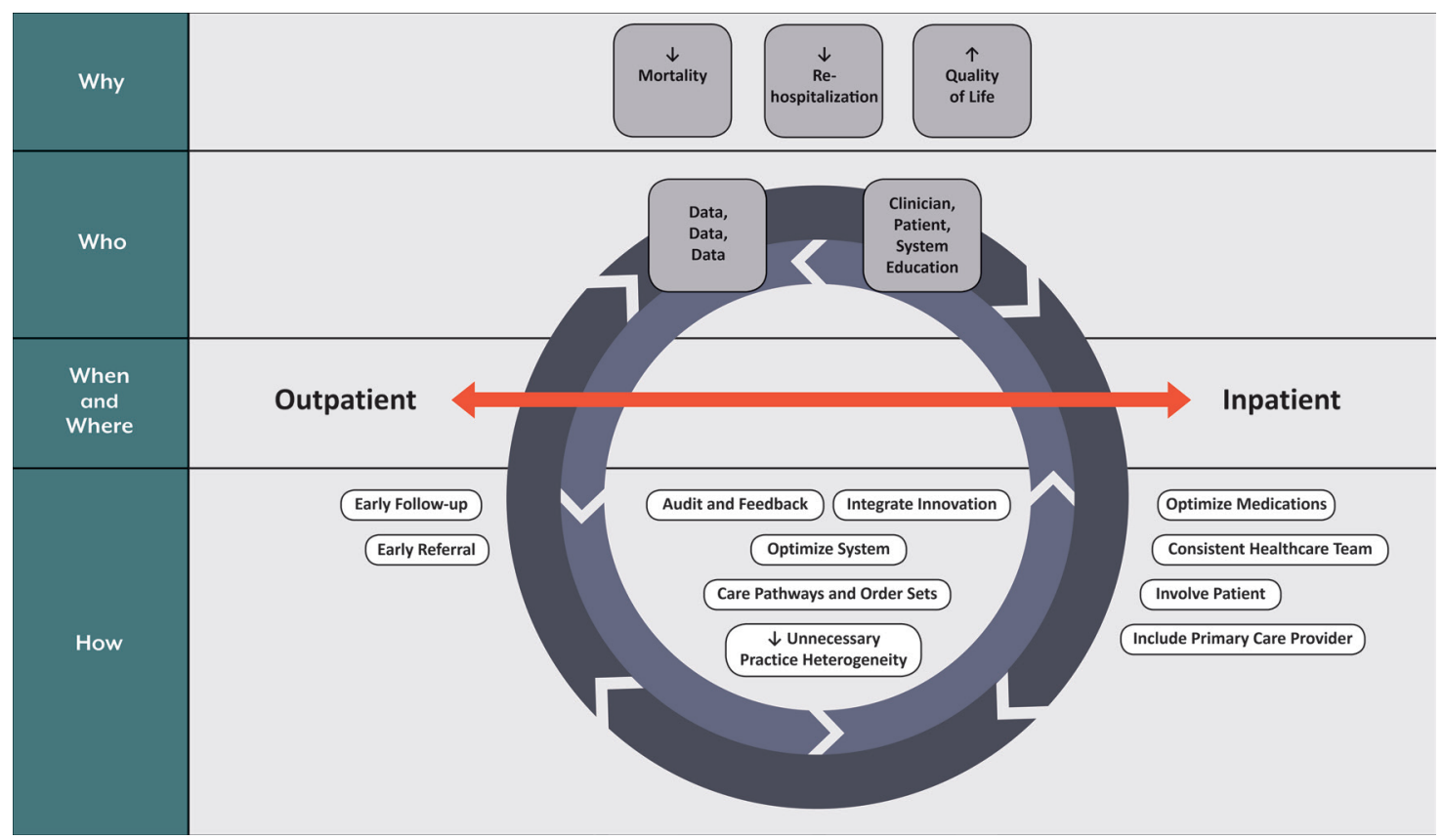

Figure 3 The complex interaction of ensuring quality improvement identifies why it is undertaking the initiative, who should be involved in developing and deploying it and when, where and how this should be done. Critically, the initiatives should move seamlessly between inpatient and outpatient environments. Examples under 'How' are suggestions, and many other ideas should be considered. 


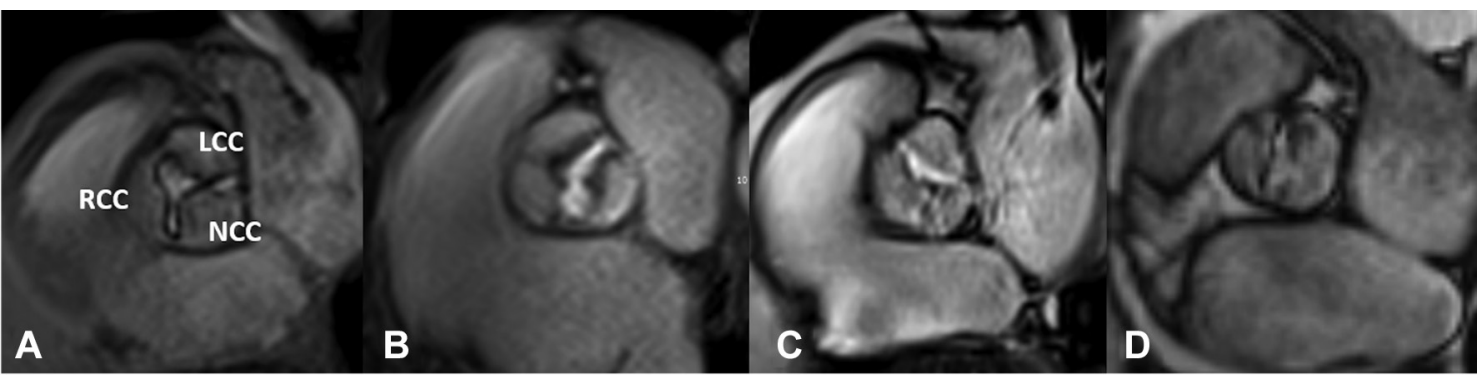

Figure 4 Morphology of aortic valve: (A) tricuspid, (B) bicuspid type-I (fusion of right and left coronary cusps), (C) bicuspid type-II (fusion of right and non-coronary cusps), (D) bicuspid type-III (fusion of left and non-coronary cusps). LCC, left coronary cusp; NCC, non-coronary cusp; RCC, right coronary cusp.

communicate the results; share, scale and spread; establish team ownership; think globally, act locally and grow the teams organically as needed.'

The Education in Heart article in this issue reviews the role of cardiac magnetic resonance imaging (CMR)in assessment of patients with aortic stenosis. ${ }^{7}$ Although echocardiography remains the key technique for diagnosis of aortic stenosis, CMR may be helpful when there are apparent unexplained discrepancies in quantitation of stenosis severity, better definition of valve morphology would be helpful or when evaluation of left ventricular fibrosis is needed. (figure 4)

Our Cardiology in Focus section has an article by Coyle and Evans ${ }^{8}$ (8) which addresses the question of why young physicians are motivated to apply for highly competitive training position in Cardiology. Young doctors perceive cardiology as interesting, prestigious and financially rewarding but also lacking diversity and characterised by adverse job conditions that interfere with family life. The authors conclude: "While the acute nature of our specialty requires out-of-hours work and procedural competencies demand many hours of practice, we must seek to find a balance so that we can continue to attract the brightest and the best.'

Funding The authors have not declared a specific grant for this research from any funding agency in the public, commercial or not-for-profit sectors.

Competing interests None declared.

Patient consent for publication Not required.

Provenance and peer review Commissioned; internally peer reviewed.

(C) Author(s) (or their employer(s)) 2019. No commercial re-use. See rights and permissions. Published by BMJ.

\section{Check for updates}

To cite Ribeiro AL, Otto CM. Heart 2019;105:423-425.
Received 5 February 2019

Accepted 5 February 2019

Heart 2019:105:423-425.

doi:10.1136/heartjnl-2019-314863

\section{REFERENCES}

1 Johansson M, Ricci F, Di Martino G, et al. Cardiovascular biomarkers predict fragility fractures in older adults. Heart 2019:105:449-54.

2 Lewis JR, Szulc P. Broken hearts and bones: new insights or falling for unmeasured confounding? Heart 2019:105:427-8.

3 Bulka CM, Daviglus ML, Persky VW, et al. Association of occupational exposures with cardiovascular disease among US Hispanics/Latinos. Heart 2019;105:439-48.

4 Broberg K. New light on exposure to chemicals and cardiovascular diseases. Heart 2019;105:426.

5 Agarwal A, Bahiru E, Yoo SGK, et al. Hospital-based quality improvement interventions for patients with heart failure: a systematic review. Heart 2019;105:431-8.

6 Ezekowitz JA. Creating order out of chaos: quality of care in heart failure. Heart 2019:105:429-30.

7 Singh A, McCann GP. Cardiac magnetic resonance imaging for the assessment of aortic stenosis. Heart 2019;105:489-97.

8 Coyle C, Evans H. A career in cardiology: why? Heart 2019;105:498. 